Pacific Journal of Mathematic 


\title{
ON THE TOPOLOGY OF DIRECT LIMITS OF ANR'S
}

\author{
R. E. HEISEY AND H. TORUŃCZYK
}

Let $\left\{\left(X_{n}, a_{n}\right)\right\}$ be a sequence of pointed, locally compact, finite-dimensional, nondegenerate, connected ANR's. It is shown that the dirct limit of the system $X_{1} \longrightarrow X_{1} \times\left\{a_{2}\right\} \subset X_{1} \times X_{2} \longrightarrow X_{1} \times X_{2} \times\left\{a_{3}\right\} \subset X_{1} \times X_{2} \times X_{3}$

is homeomorphic to an open subset of $R^{\infty}=\lim _{\rightarrow} R^{n}, R$ the reals. As a consequence, if $f: X \rightarrow Y$ is a homotopy equivalence between ANR's as above then $\lim _{\rightarrow} f^{n}: \lim _{\rightarrow} X^{n} \rightarrow \lim _{\rightarrow} Y^{n}$ is homotopic to a homeomorphism.

A. Introduction. Infinite countable products of complete AR's have been shown to be in most cases homeomorphic to either the Hilbert cube or a Hilbert space: by combined results of Anderson [1], West [9] and Edwards [2] the product $\Pi X_{i}$ is homeomorphic to $\Pi_{i}[0,1]_{i}$ provided all the $X_{i}$ are compact and nondegerate; similarly, any product of countably many noncompact AR's of the same weight is, topologically, a Hilbert space (see [8]). The latter result can be used to show that if $\left(X_{i}, a_{i}\right)$ are pointed, finite-dimensional, $\sigma$-compact AR's then the space

(i) $\sum\left(X_{i}, a_{i}\right)=\left\{\left(x_{i}\right) \in \Pi X_{i}: x_{i}=a_{i}\right.$ for almost all $\left.i\right\}$ is, in the product topology, homeomorphic to the incomplete linear subspace $l_{2}^{f}$ consisting of all eventually zero sequences in $l_{2}$, the Hilbert space.

In this note we show that, under the additional assumption that the $X_{i}$ 's are locally compact, the space (i) considered in the direct limit topology is homeomorphic to another familiar topological space, namely $R^{\infty}$, the direct limit of finite products of $R$, the real line. More generally, we have the following:

THEOREM. Let $\left\{\left(X_{n}, a_{n}\right)\right\}$ be a sequence of pointed, locally compact, finite-dimensional, connected ANR's having more than one point. Then the direct limit of the system

$$
X_{1} \longrightarrow X_{1} \times\left\{a_{2}\right\} \subset X_{1} \times X_{2} \longrightarrow X_{1} \times X_{2} \times\left\{a_{3}\right\} \subset X_{1} \times X_{2} \times X_{3}
$$

is homeomorphic to an open subset of $R^{\infty}$.

For results concerning the topological properties of $R^{\infty}$ we refer the reader to [4] and [5]. It is shown there that the $R^{\infty}$-manifolds 
posess many of the properties of $l_{2}$-manifolds; in particular, if $f$ is a homotopy equivalence between $R^{\infty}$-manifolds then $f$ is homotopic to a homeomorphism. Combined with a result of Hansen, Theorem 6.2 of [3], this gives the following.

Corollary. If $f: X \rightarrow Y$ is a homotopy equivalence betwee locally compact, finite-dimensional, connected ANR's having more than one point, then $\lim _{\rightarrow} f^{n}: \lim _{\rightarrow} X^{n} \rightarrow \lim _{\rightarrow} Y^{n}$ is homotopic to a homeomorphism.

Despite the above-mentioned similarity of $R^{\infty}$ and $l_{2}$ manifolds no intrinsic characterization of $R^{\infty}$-manifolds corresponding to the characterizations of $l_{2}$ and $Q$-manifolds (see [8]) is known. The motivation of this paper was to show that the direct limit operation leads naturally to such manifolds (see also the Proposition in $\S \mathrm{C}$ ). Earlier, it was shown by Henderson [6] that taking products of $R^{\infty}$ with locally compact, finite-dimensional ANR's yields open subsets of $R^{\infty}$. Our result generalizes Henderson's. However, while Henderson's technique involved the linear structure of $R^{\infty}$ (and has since been applied to studying factors of other linear topological spaces) our proofs involve merely the construction of embeddings from finitedimensional compacta into products of ANR's.

B. Notation and lemmas. In this section all spaces are separable and metric. If $d_{i}$ is the metric on $X_{i}, i \leqq n$, we take max $\left\{d_{i}\left(x_{i}, y_{i}\right): i \leqq n\right\}$ as the metric on $X_{1} \times \cdots \times X_{n}$. By $I$ and $I^{k}$ was denote $[0,1]$ and the $k$-fold product of $[0,1]$, respectively. If $k=0$, $I^{k}$ is the singleton.

A map (= continuous function) $g: X \rightarrow Y$ is said to be approximable by elements of the family $\mathscr{F}$ of maps $X \rightarrow Y$ if for any admissible metric $d$ for $Y$ there is an $f \in \mathscr{F}$ such that $d(f, g)<1$. (If $X$ is compact this coincides with the concept of being in the closure of $\mathscr{F}$ in the compact-open topology.)

We say that $A \subset X$ is a $Z^{k}$-set, $k \geqq 0$, if any map $I^{k} \rightarrow X$ can be approximated by maps whose images are disjoint from $A$. A map whose image is a $Z^{k}$-set will be called a $Z^{k}$-map.

We shall consider spaces $X$ having the following property, sometimes called the disjoint $k$-cube property.

$\left({ }^{*}\right)_{k} \quad$ Any map $I^{k} \times\{1,2\} \rightarrow X$ is approximable by maps sending $I^{k} \times\{1\}$ and $I^{k} \times\{2\}$ to disjoint sets.

The following generalizes the fact that $R^{2 k+1}$ has property $\left({ }^{*}\right)_{k}$.

Lemma 1. If $X_{1}, X_{2}, \cdots, X_{2 k+1}$ are locally contractible spaces with no isolated points then $X_{1} \times \cdots \times X_{2 k+1}$ has the property $(*)_{k}$. 
For a proof see [8].

Lemma 2. If $X$ is complete and satisfies $\left({ }^{*}\right)_{k}$ then any map $I^{k} \rightarrow X$ is approximable by $Z^{k}$-maps.

The proof is the same as that of Remark 3 of [7].

LEMMA 3. Let $X$ be an ANR satisfying $\left({ }^{*}\right)_{k}$, let $A$ and $B$ be disjoint compacta of dimension $\leqq k$, and let $X_{0}$ be a closed $Z^{k}$-set in $X$. Then any map $A \cup B \rightarrow X$ is approximable by maps $g: A \cup B \rightarrow X$ satisfying $g(A) \cap g(B)=\varnothing$ and $g(A \cup B) \cap X_{0}=\varnothing$.

Proof. Since $X$ is an ANR each map $A \cup B \rightarrow X$ can be approximated by compositions of the form $A \cup B \rightarrow K \rightarrow X$, where $K$ is a polyhedron of dimension $\leqq \operatorname{dim}(A \cup B)$. Thus, we may assume that $A$ and $B$ are compact polyhedra, and the result follows from the fact that in this case $A$ and $B$ are finite unions of cells of dimension $\leqq k$. (Details are left to the reader; cf. the proof of the next result.)

Proposition 4. Let $A$ and $X$ be locally compact spaces, let $A_{0}$ be a closed subset of $A$ and let $f: A \rightarrow X$ be a map such that $f\left(A_{0}\right)$ is a closed $Z^{k}$-set. If $\operatorname{dim} A \leqq k$ and $X$ is an ANR satisfying $\left({ }^{*}\right)_{k}$, then $f$ is approximable by $Z^{k}$-maps $g: A \rightarrow X$ such that $g\left|A_{0}=f\right| A_{0}$, $g\left(A \backslash A_{0}\right) \cap g\left(A_{0}\right)=\varnothing$, and $g \mid\left(A \backslash A_{0}\right)$ is one-to-one.

Proof. A proof is given in [7] for the case $k=\infty$ and $A_{0}=\varnothing$. The proof of the general case is similar; we include it for completeness.

Fix a metric $d_{0}$ for $X$. Let $d \geqq d_{0}$ be a complete metric for $X$ and let $\left\{A_{i}\right\}_{i \in N}$ be a family of compact subsets of $A \backslash A_{0}$ such that for any pair $x$ and $y$ of distinct points of $A \backslash A_{0}$ there are $i, j \in N$ with $x \in A_{i}, y \in A_{j}$, and $A_{i} \cap A_{j}=\varnothing$. Let $\left\{f_{i}\right\}_{i \in N}$ be a dence subset of $C\left(I^{k}, X\right)$ consisting of $Z^{k}$-maps such that $f_{i}\left(I^{k}\right) \cap f\left(A_{0}\right)=\varnothing$ (see Lemma 2). With $F=\left\{g \in C(A, X): g\left|A_{0}=f\right| A_{0}\right\}$ it follows from Lemma 3 and [7, Lemma C] that for each $i, j \in N$ with $A_{i} \cap A_{j}=\varnothing$, the set

$$
\begin{aligned}
G_{i, j, l}= & \left\{g \in F: g\left(A_{i}\right) \cap g\left(A_{j}\right)=\varnothing\right. \text { and } \\
& \left.g\left(A_{i} \cup A_{j}\right) \cap\left[f_{l}\left(I^{k}\right) \cup f\left(A_{0}\right)\right]=\varnothing\right\}
\end{aligned}
$$

is dense and open in $F$. (We equip $F$ with the sup metric $\hat{d}$ induced by $d$.) Since $(F, \hat{d})$ is complete it follows that $G=\cap\left\{G_{i, j, l}: A_{i} \cap A_{j}=\right.$ $\varnothing, l \in N\}$ is dense in $F$. This completes the proof since $f \in F$ and any $g \in G$ satisfies the desired conditions.

COROLLARY 5. If in Lemma 4 it is additionally assumed that 
$f$ is proper and $f \mid A_{0}$ is an embedding, then the approximations $g: A \rightarrow X$ can be taken to be closed $Z^{k}$-embeddings.

Proof. Use the facts that a map sufficiently close to a proper map of locally compact spaces is itself proper and that one-to-one proper maps are closed embeddings.

REMARK. If $A_{0}=\varnothing$ and $X=R^{2 k+1}$ then the above corollary reduces to the classical Menger-Nöbeling embedding theorem.

Lemma 6. Let $X_{1}, \cdots, X_{k}$ be nondegenerate, connected ANR's. Then the singletons are $Z^{k}$-sets in $X_{1} \times \cdots \times X_{k+1}$. Accordingly, $X_{0} \times\{b\}$ is a $Z^{k}$-set in $X_{0} \times X_{1} \times \cdots \times X_{k+1}$, for any space $X_{0}$ and any point $b \in X_{1} \times \cdots \times X_{k+1}$.

Proof. (By induction on k.) Let $b=\left(b_{1}, \cdots, b_{k+1}\right) \in X_{1} \times \cdots \times$ $X_{k+1}, f=\left(f_{1}, \cdots, f_{k+1}\right): I^{k} \rightarrow X_{1} \times \cdots \times X_{k+1}$ and $\varepsilon>0$ be given. Let $\mathscr{T}$ be a triangulation of $I^{k}$ so fine that for each simplex $\sigma \in \mathscr{T}$, $f_{k+1}(\sigma)$ is contractible in $X_{k+1}$ within a set of diameter less than $\varepsilon$. Let $\mathscr{T}^{k-1}$ be the $(k-1)$-skeleton of $\mathscr{T}$. By the induction hypothesis and [7, Lemma C] we may assume without loss of generality that $\left(f_{1}, \cdots, f_{k}\right)\left(\left|T^{k-1}\right|\right)$ misses $\left(b_{1}, \cdots, b_{k-1}\right)$; cf. proof of 4 .

Now, using the $\varepsilon$-contractions of $f_{k+1}(\sigma)$, we may alter $f$ on $k$ dimensional simplices, modulo their boundaries, so that the resulting map $g: I^{k} \rightarrow X_{1} \times \cdots \times X_{k+1}$ is within $\varepsilon$ of $f$ and satisfies (a) $g_{i}\left(I^{k}\right)=$ $f_{i}\left(I^{k}\right), i \leqq k$, and (b) for each $\sigma \in \mathscr{T} \mid \mathscr{T}^{k-1}$ there is a point $p_{o} \in X_{k+1}$ with

$$
g(\sigma) \subset\left[\left(f_{1}, \cdots, f_{k}\right)(\partial \sigma) \times X_{k+1}\right] \cup\left[\left(f_{1}, \cdots, f_{k}\right)(\sigma) \times\left\{p_{\sigma}\right\}\right] .
$$

Since $X_{k+1}$ has no isolated points, all the $p_{\sigma}$ 's can clearly be chosen distinct from $b_{k+1}$. Thus, $g$ is an $\varepsilon$-approximation to $f$ whose image misses $b$.

Finally, we need the following.

Lemma 7. Let $A$ be a locally compact space and let $A_{0}$ be a closed subset of $A$. Then any proper map $f: A_{0} \rightarrow[0,1)$ has a continuous extension $\bar{f}: A \rightarrow[0,1)$ which is also proper.

Proof. Let $A \cup\{\infty\}$ be the one point compactification of $A$, and extend $f$ to $g: A_{0} \cup\{\infty\} \rightarrow[0,1]$ by defining $g(\infty)=1$. Letting $\bar{g}: A \cup\{\infty\} \rightarrow[0,1]$ be an extension of $g$ we may take $\bar{f}(a)=h(a) \bar{g}(a)$, where $h: A \cup\{\infty\} \rightarrow[0,1]$ is a map with $h^{-1}(1)=A_{0} \cup\{\infty\}$. 
C. Proof of the theorem. The theorem follows immediately from Lemmas 1 and 6 and the following.

Proposition. Let $\left\{X_{k} ; i_{k}\right\}$ be a direct system of closed embeddings $i_{k}: X_{k} \rightarrow X_{k+1}$ of locally compact, finite-dimensional ANR's. Assume that for any positive integers $k, p$ there is an integer $l>k$ such that $X_{l}$ has property $\left({ }^{*}\right)_{p}$ and $i_{l-1} \circ \cdots \circ i_{k}\left(X_{k}\right)$ is a $Z^{p}$-set in $X_{l}$. Then, $\lim \left\{X_{k} ; i_{k}\right\}$ is homeomorphic to an open subset of $R^{\infty}$.

Proof. Let $d_{k}=\operatorname{dim} X_{k}$. Passing to a subsequence, if necessary, we may assume that

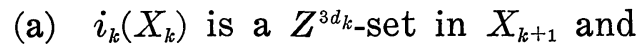

(b) $X_{k+1}$ has property $\left({ }^{*}\right)_{3 d_{k}}$ and, hence, $d_{k+1} \geqq 3 d_{k}$. Let $J=[-1, \infty)$ and let $j_{k}: J^{3 d_{k-1}} \rightarrow J^{3 d_{k-1}} \times(0,0, \cdots, 0) \subset J^{3 d_{k}}$ be the natural inclusion. We shall inductively construct manifolds with boundary $M_{k}$ in $(-1, \infty)^{3 d_{k-1}}$ and closed embeddings $f_{k}: M_{k} \rightarrow X_{k}$ and $g_{k}: X_{k} \rightarrow M_{k+1}$ such that, for each $k$,

(c) $M_{k+1}$ is a neighborhood of $j_{k}\left(M_{k}\right)$, and

(d) the following diagram commutes.

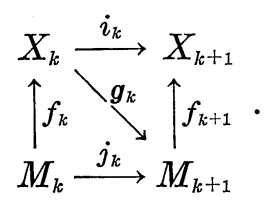

Assume $\left\{\left(M_{k}, f_{k}, g_{k}\right)\right\}$ have been constructed. It is then clear that bot $\lim \left\{X_{k} ; i_{k}\right\}$ and $\lim \left\{M_{k} ; j_{k}\right\}$ are homeomorphic to the direct limit of the system $M_{0} \stackrel{f_{0}}{\rightarrow} X_{0} \stackrel{g_{0}}{\rightarrow} M_{1} \stackrel{f_{1}}{\rightarrow} X_{1} \stackrel{g_{1}}{\rightarrow} M_{2} \stackrel{f_{2}}{\rightarrow} \cdots$. Also, it is clear that $\lim \left\{M_{k} ; j_{k}\right\}$ is homeomorphic to $\lim \left\{\operatorname{Int} M_{k} ; j_{k}\right\}$ which is open in $\overleftrightarrow{\lim }\left\{(-1, \infty)^{3 d_{k-1}} ; j_{k}\right\} \cong R^{\infty}$. Thus, $\overrightarrow{\lim }\left\{X_{k} ; i_{k}\right\}$ is homeomorphic to an $\overrightarrow{\text { open }}$ subset of $R^{\infty}$.

We now give the construction of the embeddings $f_{k}$ and $g_{k}$. Assuming, without loss of generality, that $X_{0}=R^{0}$, the singleton, we take for $f_{0}$ the identity. Having established $f_{k}$ consider the closed embedding $j_{k} f_{k}^{-1}: i m\left(f_{k}\right) \rightarrow J^{3 d_{k}}$. By Lemma 7 we can extend $j_{k} f^{-1}$ to a proper map $X_{k} \rightarrow J^{3 d_{k}}$ which we may then, by 1 and 6 , alter so as to get a closed embedding $g_{k}: X_{k} \rightarrow J^{3 d_{k}}$ coinciding with $j_{k} f_{k}^{-1}$ on $i m\left(f_{k}\right)$. Clearly, we may adjust $g_{k}$ so that in addition $i m\left(g_{k}\right) \subset(-1, \infty)^{3 d_{k}}$.

The set $i m\left(g_{k}\right)$ being a closed ANR subset of $(-1, \infty)^{3 d_{k}}$, there is a manifold with boundary $M_{k+1}$ contained in $(-1, \infty)^{3 d_{k}}$ which is topologically closed in $J^{3 d_{k}}$, contains a neighborhood of $i m\left(g_{k}\right)$ in $(-1, \infty)^{3 d_{k}}$, and which properly retracts to $i m\left(g_{k}\right)$. Then $i_{k} g_{k}^{-1}: i m\left(g_{k}\right) \rightarrow$ 
$X_{k+1}$ extends to a proper map $M_{k+1} \rightarrow X_{k+1}$ which we again may alter modulo $\mathrm{im}\left(\mathrm{g}_{k}\right)$ to get a closed embedding $f_{k+1}: M_{k+1} \rightarrow X_{k+1}$ coinciding with $i_{k} g_{k}^{-1}$ on $i m\left(g_{k}\right)$. This completes the inductive step and the proof of the proposition.

\section{REFERENCES}

1. R. D. Anderson, The Hilbert cube as a product of dendrons, Amer. Math. Soc. Notices, 11 (1964), 572.

2. T. A. Chapman, Lectures on Hilbert cube manifolds, C.B.M.S. Regional Conference Series in Math., No. 28, Amer. Math. Soc., (1976).

3. V. L. Hansen, Some theorems on direct limits of expanding sequences of manifolds, Math. Scand., 29 (1971), 5-36.

4. R. E. Heisey, Manifolds modeled on $R^{\infty}$ or bounded weak.* topologies, Trans. Amer. Math. Soc., 206 (1975), 295-312.

5. - Manifolds modeled on the direct limit of lines, (to appear in this journal).

6. D. W. Henderson, A simplicial complex whose product with any ANR is a simplicial complex, General Topology and its Applications, 3 (1973), 81-83.

7. H. Toruńckyk, On CE-images of the Hilbert cube and characterization of Q-manifolds, Fund. Math., (to appear).

8. Characterization of infinite-dimensional manifolds, Proc. Warsaw Geometric Top. Conf.

9. J. E. West, in finite products which are Hilbert cubes, Trans. Amer. Math. Soc., 150 (1970), 1-25.

Received June 15, 1979 and in revised form February 7, 1980.

VANDERBILt University

Nashville, TN 37235

AND

Institute of Math, Polish Academy of Sciences

Sniadeckich 8, 00-950 Warsaw, Poland 


\title{
PACIFIC JOURNAL OF MATHEMATICS
}

\section{EDITORS}

\author{
DoNALD BABBITT (Managing Editor) \\ University of California \\ Los Angeles, CA 90024 \\ Hugo RossI \\ University of Utah \\ Salt Lake City, UT 84112 \\ C. C. MOORE and ANDrew OGG \\ University of California \\ Berkeley, CA 94720
}

J. DugundJI

Department of Mathematics

University of Southern California

Los Angeles, CA 90007

R. FINN and J. MILGRAM

Stanford University

Stanford, CA 94305

\section{ASSOCIATE EDITORS}
R. Arens
E. F. BeCKenbaCH
B. H. NeumanN
F. WOLF
K. YoSHIDA

\section{SUPPORTING INSTITUTIONS}

UNIVERSITY OF ARIZONA

UNIVERSITY OF BRITISH COLUMBIA

CALIFORNIA INSTITUTE OF TECHNOLOGY

UNIVERSITY OF CALIFORNIA

MONTANA STATE UNIVERSITY

UNIVERSITY OF NEVADA, RENO

NEW MEXICO STATE UNIVERSITY

OREGON STATE UNIVERSITY

\author{
UNIVERSITY OF OREGON \\ UNIVERSITY OF SOUTHERN CALIFORNIA \\ STANFORD UNIVERSITY \\ UNIVERSITY OF HAWAII \\ UNIVERSITY OF TOKYO \\ UNIVERSITY OF UTAH \\ WASHINGTON STATE UNIVERSITY \\ UNIVERSITY OF WASHINGTON
}

The Supporting Institutions listed above contribute to the cost of publication of this Journal, but they are not owners or publishers and have no responsibility for its content or policies.

Mathematical papers intended for publication in the Pacific Journal of Mathematics should be in typed form or offset-reproduced, (not dittoed), double spaced with large margins. Please do not use built up fractions in the text of the manuscript. However, you may use them in the displayed equations. Underline Greek letters in red, German in green, and script in blue. The first paragraph or two must be capable of being used separately as a synopsis of the entire paper. Please propose a heading for the odd numbered pages of less than 35 characters. Manuscripts, in triplicate, may be sent to any one of the editors. Please classify according to the scheme of Math. Reviews, Index to Vol. 39. Supply name and address of author to whom proofs should be sent. All other communications should be addressed to the managing editor, or Elaine Barth, University of California, Los Angeles, California, 90024.

50 reprints to each author are provided free for each article, only if page charges have been substantially paid. Additional copies may be obtained at cost in multiples of 50 .

The Pacific Journal of Mathematics is issued monthly as of January 1966. Regular subscription rate: $\$ 102.00$ a year $(6$ Vols., 12 issues). Special rate: $\$ 51.00$ a year to individual members of supporting institutions.

Subscriptions, orders for numbers issued in the last three calendar years, and changes of address shoud be sent to Pacific Journal of Mathematics, P.O. Box 969, Carmel Valley, CA 93924, U.S.A Old back numbers obtainable from Kraus Periodicals Co., Route 100, Millwood, NY 10546.

\section{PUBLISHED BY PACIFIC JOURNAL OF MATHEMATICS, A NON-PROFIT CORPORATION}

Printed at Kokusai Bunken Insatsusha (International Academic Printing Co., Ltd.). 8-8, 3-chome, Takadanobaba, Shinjuku-ku, Tokyo 160, Japan. 


\section{Pacific Journal of Mathematics}

Vol. 93, No. $2 \quad$ April, 1981

Ilya Eugene Blum and Srinivasa Swaminathan, Continuous selections and

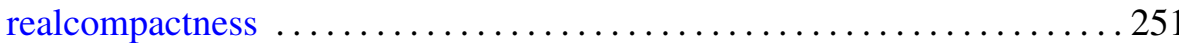

Lawrence James Brenton, Differentiably $k$-normal analytic spaces and extensions of holomorphic differential forms $\ldots \ldots \ldots \ldots \ldots \ldots \ldots 261$

Jo-Ann Deborah Cohen, Topologies on the ring of integers of a global field ................................................... 269

Robert Jay Daverman, Detecting the disjoint disks property $\ldots \ldots \ldots \ldots 277$

Edmund H. Feller, Rings where the annihilators of $\alpha$-critical modules are prime ideals ....................................... 299

Richard Elam Heisey and Henryk Torunczyk, On the topology of direct

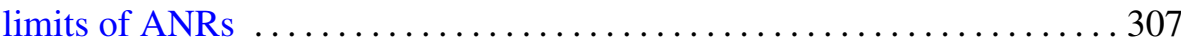

Gerald William Johnson and David Lee Skoug, Notes on the Feynman

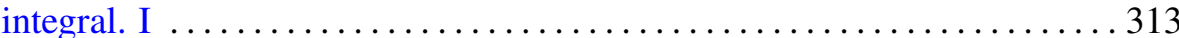

Michael S. Keane and Stuart Jay Sidney, Distinguishing a plane curve

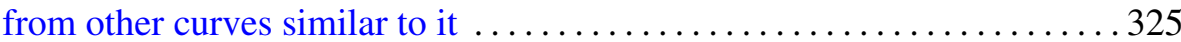

Leonid A. Luxemburg, On compact metric spaces with noncoinciding

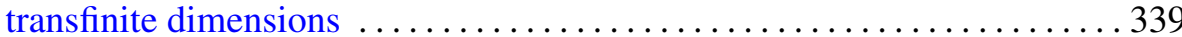

Chun Ming Ma, A uniqueness theorem for Navier-Stokes equations . . . . . . 387

Donald J. Newman and Theodore Joseph Rivlin, A characterization of the weights in a divided difference . . . . . . . . . . . . . . . . . . . 407

Marc Aristide Rieffel, $C^{*}$-algebras associated with irrational rotations $\ldots .415$

Kichi-Suke Saito, Invariant subspaces for finite maximal subdiagonal algebras

Frederic W. Shultz, Dual maps of Jordan homomorphisms and ${ }^{*}$-homomorphisms between $C^{*}$-algebras $\ldots \ldots \ldots \ldots$

Vsevolod Alekseevich Solonnikov, On the solvability of boundary and initial-boundary value problems for the Navier-Stokes system in domains with noncompact boundaries

Tavan Thomas Trent, New conditions for subnormality

L. E. Ward, Extending Whitney maps ..................

Leslie Wilson, Jets with regular zeros

Sergio Eduardo Zarantonello, The sheaf of $H^{p}$-functions in product 